\title{
Tamanho e forma de parcela em experimentos com morangueiro cultivado em solo ou em hidroponia
}

\author{
Carine Cocco(1) $^{(1)}$ Alexandra Augusti Boligon ${ }^{(1)}$, Jerônimo Luis Andriolo(1), Clarisse Silva Oliveira(1) \\ e Leandro Homrich Lorentz ${ }^{(2)}$
}

\begin{abstract}
(1)Universidade Federal de Santa Maria, Departamento de Fitotecnia, Avenida Roraima s/no, Bairro Camobi, CEP $97105-900$ Santa Maria, RS E-mail: carinecocco@yahoo.com.br, aboligon@yahoo.com.br, andriolo@smail.ufsm.br, clarisoliveira@yahoo.com.br (2)Universidade de Santa Catarina, Centro de Educação Superior do Oeste, Rua Benjamin Constant, oㅡ 84 E, CEP 89802-200 Chapecó, SC. E-mail: leandrolorentz@udesc.br
\end{abstract}

Resumo - O objetivo deste trabalho foi estimar a forma e o tamanho de parcela ótimos para ensaios com a cultura do morangueiro (Fragaria x ananassa) em cultivo hidropônico e em solo. Foram conduzidos dois, experimentos, um em cultivo convencional no solo, em túneis baixos, e outro em cultivo hidropônico. Em cada experimento, avaliaram-se os efeitos do tamanho e do formato das parcelas sobre a precisão experimental. Cada planta foi considerada uma unidade básica, e o número de unidades básicas por parcela variou de 1 (48 parcelas) a 24 (duas parcelas). Foram ajustadas funções para a determinação do coeficiente de variação entre as parcelas e para a determinação da variância por unidade básica entre as parcelas. O cultivo no solo apresentou maior variabilidade experimental que o cultivo hidropônico. $\mathrm{O}$ aumento no número de plantas por parcela causou redução acentuada na variabilidade experimental, especialmente quando se usou o formato de parcela retangular. O tamanho ótimo estimado das parcelas é de dez plantas, no cultivo com solo, e de seis plantas, no cultivo hidropônico.

Termos para indexação: Fragaria x ananassa, coeficiente de variação, índice de heterogeneidade do solo, presisão experimental.

\section{Plot size and shape in trials using strawberry cultivated with soil or using hydroponics}

\begin{abstract}
The objective of this work was to estimate the optimal size and shape of plots to be used in experiments of strawberry (Fragaria $\mathrm{x}$ ananassa) cultivation in soil or using hydroponics. Two experiments were conducted, one in soil in low tunnels, and another in a hydroponic system. In each experiment, the effects of plot sizes and shapes on experimental accuracy were evaluated. Each plant was considered an experimental basic unit, and the number of plants per plot varied from 1 (48 plots) to 24 (two plots). Functions were adjusted to determine the coefficient of variation among plots and the variance per basic unit between plots. Plants grown in soil had higher experimental variability than the plants grown in hydroponics. Increasing the number of plants per plot caused strong reduction in the experimental variability, especially when a rectangular plot shape was used. The optimal estimated plot size was ten plants in soil and six plants in the hydroponic system.

Index terms: Fragaria $\mathrm{x}$ ananassa, coefficient of variation, soil heterogeneity index, experimental accuracy.
\end{abstract}

\section{Introdução}

O morangueiro tem seu cultivo tradicional no campo, geralmente em canteiros cobertos por plástico preto (Furlani, 2001). Entretanto, o cultivo hidropônico vem se expandindo nos últimos anos. Segundo Giménez et al. (2008), essa expansão ocorre principalmente pelas dificuldades em controlar as doenças e pragas do solo, decorrentes da proibição do uso do brometo de metila. Esses autores destacam o aumento, nos últimos anos, do número de publicações sobre a produção do morangueiro em sistema hidropônico.
O sistema de cultivo hidropônico caracterizase por maior controle das variáveis ambientais, quando comparado ao de cultivo no solo (Andriolo, 1999). Ao planejar experimentos, deve-se levar em consideração as características de cada sistema de produção, para a obtenção de resultados confiáveis. Uma das técnicas para minimizar o erro experimental e aumentar a precisão dos experimentos consiste na utilização de parcelas com forma e tamanho adequados (Storck et al., 2005, 2006). A parcela, unidade básica do experimento, deve ser capaz de reduzir ao máximo o efeito da heterogeneidade 
ambiental e da variabilidade genética do material experimental (Freitas et al., 2001). A diminuição do erro experimental tem como objetivo aumentar o poder dos testes de comparação de médias, por meio da redução no intervalo de confiança entre as médias.

A variabilidade em uma área experimental é dependente de vários fatores, como: natureza do material experimental, heterogeneidade do solo, número de repetições, número de tratamentos $\mathrm{e}$ competição entre plantas (Bertolucci et al., 1991). A determinação do tamanho ótimo das parcelas deve ser feita para cada condição de cultivo e não pode ser generalizada para outras situações. Os trabalhos destinados a determinar o tamanho e a forma das parcelas são constituídos, em sua grande maioria, por experimentos de uniformidade, ou ensaio em branco (Lúcio et al., 2004). Esses experimentos também são utilizados com outros propósitos, entre os quais determinar o número de repetições, a heterogeneidade do solo, a eficiência relativa de determinado delineamento experimental e os ajustes no rendimento de experimentos subsequentes. A variável para a qual se quer determinar o tamanho ótimo das parcelas é medida separadamente, em cada unidade básica e, mediante a soma de unidades contíguas, são simulados e testados diferentes tamanhos e formas de parcelas.

Entre os métodos de determinação do tamanho ótimo das parcelas, destaca-se o método proposto por Hatheway (Storck et al., 2006), que permite estabelecer o tamanho da parcela para uma determinada diferença entre as médias que o experimentador quer detectar. Permite, ainda, conhecer a priori a diferença que poderá ser detectada entre médias para um determinado tamanho de parcela (Nagai et al., 1978).

$\mathrm{Na}$ literatura, são encontrados trabalhos que determinam o tamanho ótimo de parcela para algumas hortaliças em cultivo protegido como o tomateiro (Lopes et al., 1998), a alface em cultivo hidropônico (Marodim et al., 2000), a abobrinha italiana (Mello et al., 2004) e o pimentão (Lúcio et al., 2004). Trabalhos similares para o cultivo do morangueiro são escassos, e a metodologia utilizada na determinação do tamanho ótimo da parcela para essa cultura tem sido definida com base em resultados obtidos com outras hortaliças ou em função da área experimental disponível.

O objetivo deste trabalho foi estimar a forma e o tamanho ótimos de parcela, para ensaios com a cultura do morangueiro, conduzidos no solo e em hidroponia.

\section{Material e Métodos}

Os ensaios foram conduzidos na área experimental do Departamento de Fitotecnia, da Universidade Federal de Santa Maria, RS (29 $43^{\prime} 23^{\prime \prime S}, 53^{\circ} 43^{\prime} 15^{\prime \prime} \mathrm{W}$ e altitude de $95 \mathrm{~m}$ ). O clima da região é classificado como Cfa, subtropical úmido, sem estação seca e com verões quentes, e o solo do local é classificado como Brunizem hidromórfico (Santos et al., 2006). Foram conduzidos dois experimentos, um em cultivo convencional no solo, em túneis baixos, e outro em cultivo hidropônico. Os túneis baixos foram instalados sobre canteiros com 1,10 m de largura, cobertos com filme de polietileno transparente de $100 \mu \mathrm{m}$ de espessura. O cultivo hidropônico foi feito no interior de um abrigo, com $20 \mathrm{~m}$ de comprimento e $5 \mathrm{~m}$ de largura, coberto com filme de polietileno transparente de baixa densidade (Pebd) com $150 \mu \mathrm{m}$ de espessura.

$\mathrm{O}$ sistema hidropônico utilizado foi formado por um leito de cultivo com substrato de areia, semelhante a um canteiro, no qual cada unidade do sistema teve dimensões de 3,6 m de comprimento e 1,10 $\mathrm{m}$ de largura (Andriolo, 2007). A solução nutritiva empregada foi descrita por Furlani \& Fernandes Júnior (2004), fornecida por subirrigação, com $\mathrm{pH}$ mantido entre 5,5 e 6,5 e condutividade elétrica (CE) entre 1,4 e $1,5 \mathrm{dS} \mathrm{m}^{-1}$, com o manejo da fertirrigação e da cultura feitos de acordo com as indicações desses autores. No cultivo convencional, o preparo do solo, a adubação e o manejo da cultura foram realizados de acordo com as recomendações de Santos \& Medeiros (2003).

Em ambos os ambientes de cultivo, a superfície foi coberta com "mulching" de polietileno preto de $30 \mu \mathrm{m}$ de espessura e o plantio foi feito em quatro fileiras, em zigue-zague, com densidade de 6,6 plantas $\mathrm{m}^{-2}$ no cultivo no solo e de 12 plantas $\mathrm{m}^{-2}$ no sistema hidropônico. Foi empregado o clone SM-INIA LBD 15.1, com mudas provenientes de micropropagação produzidas no Laboratório de Melhoramento e Propagação Vegetativa de Plantas do Departamento supracitado. O plantio das mudas foi realizado em 27 de abril de 2007, e o experimento foi encerrado em 17 de dezembro de 2007. Em cada ambiente, foram utilizadas duas bancadas, com 48 plantas em cada uma. As plantas dentro de cada bancada foram numeradas em ordem crescente $\mathrm{e}$ distribuídas em quatro fileiras de 12 plantas, em área de $9,6 \mathrm{~m}^{2}$, no cultivo em solo, e de $3,6 \mathrm{~m}^{2}$ no cultivo hidropônico. 
Cada planta foi considerada uma unidade básica, e a variável avaliada foi a massa de matéria fresca total de frutas por planta, dada pela soma das produções de cada planta em cada colheita. O ponto de colheita foi caracterizado quando o fruto apresentou a epiderme totalmente vermelha, correspondente ao estádio fenológico 87 (Meier et al., 1994).

As produções individuais de cada uma das 48 unidades básicas, em cada uma das bancadas, nos dois ambientes, foram utilizadas para simular diferentes tamanhos de parcela, formadas por $\mathrm{X}_{1}$ unidades básicas de comprimento (colunas) e $\mathrm{X}_{2}$ unidades básicas de largura (linhas). Os tamanhos de parcela, simulados pelo agrupamento das produções totais das unidades contíguas de modo que $\mathrm{X}_{1} \times \mathrm{X}_{2}$ correspondesse a $\mathrm{X}$ (tamanho da parcela em número de unidades básicas), foram: 1x1, 1x2, 1x3,1x4, 1x6, 1x12, 2x3, 2x4 e $2 \times 6$. Foram calculados: número de parcelas $(\mathrm{N})$, com $\mathrm{X}$ unidades básicas (UB) de tamanho, por meio da expressão $\mathrm{N}=48 / \mathrm{X}$; média das parcelas $\left[\mathrm{M}_{(\mathrm{x})}\right]$, com $\mathrm{X}$ UB de tamanho; variância entre parcelas $\left[\mathrm{V}_{(\mathrm{x})}\right] \operatorname{com} \mathrm{X}$ UB de tamanho; variância por UB [VU $\left.\mathrm{V}_{(\mathrm{x})}\right]$, calculada entre as parcelas com X UB, por meio da expressão $\mathrm{V}_{(\mathrm{x})} / \mathrm{X}^{2}$; e coeficiente de variação entre as parcelas $\left[\mathrm{CV}_{(\mathrm{x})}\right]$ com X UB de tamanho.

Foram simulados 16 tipos diferentes de parcelas (Tabela 1), com o objetivo de variar, além tamanho das parcelas, expresso em termos de número de plantas (UBs), o número de plantas utilizado no sentido do comprimento ou da largura, para obtenção de diferentes formas de parcela. A menor parcela foi composta por uma unidade básica (uma planta), e a maior por $24 \mathrm{UB}$, e este tamanho foi utilizado em duas formas diferentes de parcela, $12 \times 2$ plantas e $6 \times 4$ plantas (comprimento $\mathrm{x}$ largura).
$\mathrm{O}$ índice de heterogeneidade do solo (b) foi estimado para cada bancada, em cada ambiente, por meio da linearização da equação $\mathrm{VU}_{(\mathrm{x})}=\mathrm{V}_{1} / \mathrm{X}^{\mathrm{b}}$, em que: $\mathrm{b}$ é o índice de heterogeneidade; $V_{1}$ é a variância entre as parcelas de uma unidade básica; e $\mathrm{X}$ é o número de unidades básicas utililizado. Como os valores de $\mathrm{VU}(\mathrm{X})$ tiveram diferentes graus de liberdade, foi realizada a ponderação das estimativas de $V_{1}$ e $b$ com base em seus respectivos números de graus de liberdade, com auxílio do programa estatístico NTIA (Embrapa, 1997). De forma análoga, foram feitas as estimativas da equação $\mathrm{CV}_{(\mathrm{x})}=\mathrm{A} / \mathrm{X}^{\mathrm{B}}$, em que: $\mathrm{A}$ representa o coeficiente de variação para parcelas de uma unidade básica e $B=b / 2$. Com base nessas determinações, e de acordo com trabalho de Lúcio et al. (2004), foi estimado o tamanho ótimo da parcela experimental, em unidades básicas, para a cultura do morangueiro, por meio da expressão abaixo: $\mathrm{X}_{0}=\exp \left\{[1 /(2 \mathrm{~B}+2)] \ln \left[\left(\mathrm{A}^{2} \mathrm{~B}^{2}(2 \mathrm{~B}+1)\right] /(\mathrm{B}+2)\right\}\right.$

Pelo método de Hatheway (Hatheway, 1961), foi calculada a estimativa da diferença entre médias de tratamentos (D), dada em percentagem da média, pela expressão:

$\mathrm{D}=\left[2\left(\mathrm{t}_{1}+\mathrm{t}_{2}\right)^{2} \mathrm{~A}^{2} / \mathrm{rX}_{0}^{\mathrm{b}}\right]^{0,5}$, em que: $\mathrm{r}$ é o número de repetições simulado; $\mathrm{X}_{0}$ é o tamanho ótimo de parcela, em número de plantas, obtido pela expressão anterior; A e b são estimativas obtidas nas expressões anteriores; $\mathrm{t}_{1}$ é o valor crítico da distribuição de $\mathrm{t}$, no nível de significância de 5\% (bilateral); e t $\mathrm{t}_{2}$ é o valor crítico da distribuição de $t$ correspondente a um erro de $2(1-p)$, considerando-se $\mathrm{p}=0,8$ (probabilidade de obter resultados significativos).

Os valores críticos da distribuição de $t$ foram obtidos de acordo com o número de graus de liberdade do resíduo. Foi considerado um experimento conduzido

Tabela 1. Tamanho de parcelas, em unidades básicas, e número total de parcelas estabelecidas no ensaio em branco de acordo com o número de unidades básicas (UB) utilizadas no sentido da largura e no comprimento, nos experimentos para a determinação do tamanho ótimo de parcelas para morangueiro, no cultivo em solo e hidropônico.

\begin{tabular}{|c|c|c|c|c|c|c|}
\hline \multirow{3}{*}{$\begin{array}{l}\text { UB no sentido } \\
\text { da largura }\end{array}$} & \multicolumn{6}{|c|}{ UB no sentido do comprimento } \\
\hline & \multicolumn{2}{|c|}{1} & \multicolumn{2}{|c|}{2} & \multicolumn{2}{|c|}{4} \\
\hline & $\begin{array}{r}\text { Tamanho de } \\
\text { parcela (UB) }\end{array}$ & $\begin{array}{l}\text { Número de } \\
\text { parcelas }\end{array}$ & $\begin{array}{r}\text { Tamanho de } \\
\text { parcela (UB) }\end{array}$ & $\begin{array}{l}\text { Número de } \\
\text { parcelas }\end{array}$ & $\begin{array}{l}\text { Tamanho de } \\
\text { parcela (UB) }\end{array}$ & $\begin{array}{c}\text { Número de } \\
\text { parcelas }\end{array}$ \\
\hline 1 & 1 & 48 & 2 & 24 & 4 & 12 \\
\hline 2 & 2 & 24 & 4 & 12 & 8 & 6 \\
\hline 3 & 3 & 16 & - & - & 12 & 4 \\
\hline 4 & 4 & 12 & 8 & 8 & - & - \\
\hline 6 & 6 & 8 & 12 & 4 & 6 & 8 \\
\hline 12 & 12 & 4 & 24 & 2 & 24 & 2 \\
\hline
\end{tabular}


no delineamento inteiramente casualizado, e foram simuladas situações em que os números de tratamentos foram 2, 4 e 8 , em combinação com os números de repetições de 2,4 e 8 .

\section{Resultados e Discussão}

O coeficiente de variação (CV) em cada tipo de parcela simulada, nas duas bancadas de plantas, em cultivo no solo e hidropônico, diminuiu com o aumento do tamanho da parcela, principalmente se esse aumento promoveu a formação de parcelas retangulares (Tabela 2). Considerando-se parcelas com largura de uma unidade básica no cultivo hidropônico, observou-se que o valor do $\mathrm{CV}$ variou de $22,97 \%$, quando se utilizou também uma UB no sentido do comprimento, a 2,78\%, quando se utilizaram 12 UB nesse sentido. Para o cultivo no solo, com a mesma largura, o valor do CV variou de 41,99 a $8,47 \%$, em parcelas com 1 e 12 UB de comprimento, respectivamente. Observou-se que o conjunto de dados foi adequado ao estudo proposto, pois o comportamento do CV em relação ao tamanho das parcelas está de acordo com outros estudos dessa natureza (Viana et al., 2002; Storck et al., 2005). A utilização de parcelas de tamanho reduzido resultou em aumento do $\mathrm{CV}$ e, consequentemente, do erro experimental, o que afetou diretamente as análises estatísticas posteriores. Também houve variação nos valores do $\mathrm{CV}$ entre os dois ambientes, e o valor máximo encontrado no cultivo em solo foi superior ao máximo encontrado no cultivo hidropônico (Tabela 2). Da mesma maneira, o valor mínimo encontrado no cultivo hidropônico foi inferior ao encontrado no cultivo no solo.

Em alguns casos, a redução do coeficiente de variação em parcelas retangulares ocorreu de maneira mais pronunciada quando essas parcelas foram simuladas no sentido do comprimento (Tabela 2). Assim, para parcelas com quatro UB, por exemplo, o CV passou de 17,15 para $8,37 \%$, no cultivo hidropônico (bancada 2), e de 26,96 para $22,10 \%$ no cultivo no solo (bancada 1 ). Nas demais situações, o CV foi muito próximo nas parcelas simuladas com as diferentes formas, e passou de 11,38 para $10,18 \%$ no cultivo hidropônico (bancada 1) e de 19,45 para
$18,86 \%$ no cultivo com solo (bancada 2 ), para parcelas retangulares no sentido da largura e do comprimento, respectivamente.

$\mathrm{O}$ menor $\mathrm{CV}$ encontrado em parcelas retangulares indica menor variabilidade dentro da linha, em comparação com a variação entre linhas de cultivo. Esse resultado está de acordo com resultados encontrados por Igue et al. (1991), que mencionam a existência de uma predominância de parcelas retangulares, com maior dimensão no comprimento da linha, visto que há maior variabilidade entre linhas que dentro delas. Para a cultura do pimentão cultivado em estufa de plástico, Lúcio et al. (2004) também encontraram parcelas retangulares no sentido da linha e recomendaram parcelas de dez plantas, com duas linhas e cinco plantas em cada linha.

A produção total de frutas por planta não diferiu entre as bancadas (Tabela 3). Porém, a mesma produção foi significativamente maior no cultivo hidropônico, em que a produção por planta foi, em média, 24\% superior à do cultivo no solo. O cultivo no solo apresentou variabilidade superior à do cultivo hidropônico, o que está refletido no desvio-padrão, na variância entre parcelas de uma unidade básica e no coeficiente A. A maior fonte de variabilidade em áreas experimentais é normalmente atribuída ao solo (Lorentz et al., 2004), fator que está ausente no cultivo hidropônico. Esse fator tem reflexo no tamanho ótimo de parcela, que no ambiente hidropônico variou de três a seis plantas. Para o cultivo no solo, o tamanho ótimo

Tabela 2. Coeficiente de variação (\%) das parcelas de diferentes tamanhos, formadas pela combinação de $\mathrm{X}$ unidades básicas (UB) no comprimento e Y UB na largura, nas duas bancadas em cada ambiente de cultivo ${ }^{(1)}$.

\begin{tabular}{|c|c|c|c|c|c|c|}
\hline \multirow[t]{2}{*}{$\begin{array}{l}\text { UB usadas no } \\
\text { comprimento }\end{array}$} & \multicolumn{3}{|c|}{$\begin{array}{c}\text { UB usadas na largura, } \\
\text { na bancada } 1\end{array}$} & \multicolumn{3}{|c|}{$\begin{array}{l}\text { UB usadas na largura, } \\
\text { na bancada } 2\end{array}$} \\
\hline & 1 & 2 & 4 & 1 & 2 & 4 \\
\hline & \multicolumn{6}{|c|}{ Cultivo hidropônico } \\
\hline 1 & 22,97 & 14,69 & 10,18 & 22,90 & 26,89 & 17,15 \\
\hline 2 & 18,40 & 13,24 & 7,87 & 13,20 & 10,84 & 9,81 \\
\hline 3 & 15,48 & 10,57 & 8,98 & 10,36 & 7,60 & 7,20 \\
\hline 4 & 11,38 & 9,29 & - & 8,37 & 4,62 & - \\
\hline 6 & 9,99 & 7,20 & 0,22 & 13,99 & 3,16 & 1,93 \\
\hline 12 & 9,47 & 8,66 & - & 2,78 & 3,29 & - \\
\hline & \multicolumn{6}{|c|}{ Cultivo no solo } \\
\hline 1 & 38,93 & 29,54 & 26,96 & 41,99 & 33,55 & 18,86 \\
\hline 2 & 26,28 & 20,29 & 21,04 & 31,45 & 22,48 & 15,70 \\
\hline 3 & 25,16 & 19,57 & 18,17 & 22,03 & 20,90 & 8,09 \\
\hline 4 & 22,10 & 18,81 & - & 19,43 & 13,63 & - \\
\hline 6 & 22,19 & 18,90 & 20,62 & 22,51 & 17,32 & 5,92 \\
\hline 12 & 12,73 & 9,87 & - & 8,47 & 4,98 & - \\
\hline
\end{tabular}


Tabela 3. Massa de matéria fresca média de frutas por planta, desvio-padrão (s), variância entre parcelas de uma unidade básica $\left(\mathrm{V}_{1}\right)$, índice de heterogeneidade do solo $(\mathrm{b})$, coeficientes $\mathrm{A}$ e $\mathrm{B}$, coeficiente de determinação $\left(\mathrm{R}^{2}\right)$ e tamanho ótimo de parcela, para a produção de frutas de morangueiro, em cultivo no solo e hidropônico ${ }^{(1)}$.

\begin{tabular}{|c|c|c|c|c|c|c|c|c|}
\hline Bancada & $\begin{array}{c}\text { Massa de } \\
\text { matéria fresca }(\mathrm{g})\end{array}$ & $\begin{array}{l}\text { Desvio- } \\
\text { padrão }\end{array}$ & $\mathrm{V}_{1}$ & $\mathrm{~b}$ & $\mathrm{~A}$ & B & $\mathrm{R}^{2}$ & $\begin{array}{l}\text { Tamanho ótimo de } \\
\text { parcela (plantas) }\end{array}$ \\
\hline & \multicolumn{8}{|c|}{ Cultivo hidropônico } \\
\hline 1 & $332,63 a$ & 76,39 & $2.474,0209$ & 0,3982 & 14,9691 & 0,1991 & 0,53 & 3 \\
\hline \multirow[t]{2}{*}{ Média } & $325,83 \mathrm{~A}$ & 74,79 & $4.742,1051$ & 0,8809 & 21,6096 & 0,4519 & - & - \\
\hline & \multicolumn{8}{|c|}{ Cultivo no solo } \\
\hline 1 & $258,91 \mathrm{a}$ & 100,80 & $2.944,2223$ & 0,9315 & 47,5499 & 0,4676 & 0,83 & 8 \\
\hline 2 & $238,10 \mathrm{a}$ & 99,98 & $28.259,9249$ & 1,6031 & 76,6155 & 0,8269 & 0,82 & 10 \\
\hline Média & $248,51 \mathrm{~B}$ & 100,39 & $15.602,0736$ & 1,2673 & 62,0827 & 0,6473 & - & - \\
\hline Média geral & 287,17 & 87,59 & $10.172,0893$ & 1,0741 & 41,8462 & 0,5346 & - & - \\
\hline
\end{tabular}

de parcela variou de oito a dez plantas. A variação no tamanho ótimo de parcela, calculado dentro de cada ambiente, pode ser atribuída à variação do índice de heterogeneidade do solo e do coeficiente de variação, que foram distintos entre as bancadas dentro de cada ambiente. Esse índice apresentou valores próximos e superiores a um na maioria das situações, o que indica que a área experimental era heterogênea, exceto na primeira bancada hidropônica. Isso mostra que parcelas adjacentes são ambientes diferentes. $\mathrm{O}$ aumento do tamanho das parcelas visa a diluir as diferenças entre as parcelas, a fim de que parcelas adjacentes sejam mais homogêneas. Porém, nessas condições há maiores dificuldades para o trabalho e os custos são aumentados. $\mathrm{Na}$ determinação do tamanho ótimo de parcelas de morangueiro, Nagai et al. (1978) encontraram índice de heterogeneidade do solo em torno de 0,2214 , muito abaixo do encontrado no presente estudo. Além disso, concluíram que, para parcelas constituídas de até seis unidades básicas, a forma tem pouca influência sobre a variância entre parcelas, o que contraria os resultados aqui encontrados.

Foi realizada a simulação de um novo experimento na área, com os tamanhos de parcela e valores de $b$ e A encontrados nos dois ambientes, tendo-se fixado diferentes números de tratamentos e de repetições. Aestimativa da diferença entre médias de tratamento(D) foi maior no cultivo em solo e variou de 20,57 a 46,11\%, enquanto a variação no cultivo hidropônico foi de 13,99 a 31,36\% (Tabela 4). Esses resultados evidenciam, novamente, a maior variabilidade encontrada em cultivo no solo e, portanto, a necessidade de que ocorra maior diferença entre médias de tratamentos, para que sejam considerados estatisticamente diferentes.
Tabela 4. Estimativas para a diferença verdadeira entre duas médias de tratamentos, expressas em percentagem da média (D\%), para diferentes combinações de número de tratamentos e número de repetições, em experimento com morangueiro em cultivo no solo e hidropônico.

\begin{tabular}{cccc}
\hline Número de repetições & \multicolumn{3}{c}{ Número de tratamentos } \\
\cline { 2 - 4 } & 2 & 4 & 8 \\
\hline & \multicolumn{3}{c}{ Cultivo no solo } \\
4 & - & - & 46,11 \\
8 & 21,74 & 31,14 & 29,81 \\
2 & - & 20,94 & 20,57 \\
4 & - & - & 31,36 \\
8 & 14,79 & 21,18 & 20,27 \\
\hline
\end{tabular}

\section{Conclusões}

1. A variabilidade experimental é acentuadamente reduzida com o aumento no número de plantas por parcela, especialmente quando se usa o formato de parcela retangular.

2. O tamanho ótimo estimado da parcela é de dez plantas em cultivo no solo e de seis plantas em cultivo hidropônico.

\section{Agradecimentos}

Ao Conselho Nacional de Desenvolvimento Científico e Tecnológico, pelo apoio financeiro; à Coordenação de Aperfeiçoamento de Pessoal de Nível Superior, pelas bolsas concedidas.

\section{Referências}

ANDRIOLO, J.L. Fisiologia das culturas protegidas. Santa Maria: UFSM, 1999. 142p. 
ANDRIOLO, J.L. Sistemas hidropônicos para a produção de frutas do morangueiro. In: SEMINÁRIO SOBRE O CULTIVO HIDRÔPONICO DE MORANGUEIRO, 1., 2007, Santa Maria. Anais. Santa Maria: UFSM, 2007. p.30-40.

BERTOLUCCI, F.L.G.; RAMALHO, M.A.P.; DUARTE, G.S. Alternativas de tamanho e forma da parcela para avaliação de progênies do feijoeiro (Phaseolus vulgaris L.). Ciência e Prática, v.15, p.295-305, 1991.

EMBRAPA. Centro Nacional de Pesquisa Tecnológica em Informática para a Agricultura. Ambiente de software NTIA. Versão 4.2.2. Campinas: Centro Nacional de Pesquisa Tecnológica em Informática para a Agricultura, 1997. 258p.

FREITAS, J.A. de; SILVA, E. de B.; FALLIERI, J.; LANZA, M.A.; FARIA, R.S. de; SILVA, P.J. da. Tamanho de amostra na parcela para caracterização da altura de plantas de algodoeiro herbáceo (Gossypium hirsutum). Ciência Rural, v.31, p.583-587, 2001.

FURLANI, P.R. Hidroponia vertical: nova opção para produção de morango no Brasil. O Agronômico, v.53, p.26-28, 2001.

FURLANI, P.R.; FERNANDES JÚNIOR, F. Cultivo hidropônico de morango em ambiente protegido. In: SIMPÓSIO NACIONAL DO MORANGO, 2.; ENCONTRO DE PEQUENAS FRUTAS E FRUTAS NATIVAS, 1., 2004, Pelotas. Anais. Pelotas: Embrapa Clima Temperado, 2004. p.102-115. (Embrapa Clima Temperado. Documentos, 124).

GIMÉNEZ, G.; ANDRIOLO, J.; GODOI, R. Cultivo sem solo do morangueiro. Ciência Rural, v.38, p.273-279, 2008.

HATHEWAY, W.H. Convenient plot size. Agronomy Journal, v.53, p.279-280. 1961.

IGUE, T.; ESPIRONELO, A.; CANTARELLA, H.; NELLI, E.J. Tamanho e forma de parcela experimental para cana-de-açúcar. Bragantia, v.50, p.163-180, 1991.

LOPES, S.J.; STORCK, L.; HELDWEIN, A.B.; FEIJÓ, S.; ROS, C.A. da. Técnicas experimentais para tomateiro tipo salada sob estufas plásticas. Ciência Rural, v.28, p.193-197, 1998.

LORENTZ, L.H.; LÚCIO, A.D.; STORCK, L.; LOPES, S.J.; BOLIGON, A.A.; CARPES, R.H. Variação temporal do tamanho de amostra para experimentos em estufa plástica. Ciência Rural, v.34, p.1043-1049, 2004.

LÚCIO, A.D.; MELLO, R.M.; STORCK, L.; CARPES, R.H.; BOLIGON, A.A.; ZANARDO, B. Estimativa de parâmetros para o planejamento de experimentos com a cultura do pimentão em área restrita. Horticultura Brasileira, v.22, p.766-770, 2004.

MARODIM, V.S.; STORCK, L.; LOPES, S.J.; SANTOS, O.S. dos; SCHIMIDT, D. Delineamento experimental e tamanho de amostra para alface cultivada em hidroponia. Ciência Rural, v.30, p.779-781, 2000.

MEIER, U.; GRAF, H.; HACK, M.; HESS, M.; KENNEL, W.; KLOSE, R.; MAPPES, D.; SEIPP, D.; STAUSS, R.; STREIF, J.; VAN DEN BOOM, D. Phänologische Entwick lungsstadien des Kernobstes (Malus domestica Borkh. und Pyrus communis L.), des Steinobstes (Prunus-Arten), der Johannisbeere (Ribes-Arten) und der Erdbeere (Fragaria $\mathrm{x}$ ananassa Duch.). Nachrichtenblatt Deutscher Pflanzenschutzdienst, v.46, p.141-153, 1994.

MELLO, R.M.; LÚCIO, A.D.; STORCK, L.; LORENTZ, L.H.; CARPES, R.H.; BOLIGON, A.A. Size and form of plots for the culture of the Italian pumpkin in plastic greenhouse. Scientia Agricola, v.61, p.457-461, 2004.

NAGAI, V.; PASSOS, F.A.; SCARANARI, H.J.; MARTINS, F.P. Tamanho da parcela e número de repetições em experimentos com morangueiro. Bragantia, v.37, p.71-81, 1978.

SANTOS, A.M. dos; MEDEIROS, A.R.M. de (Ed.). Morango: produção. Pelotas: Embrapa Clima Temperado; Brasília: Embrapa Informação Tecnológica, 2003. 81p. (Frutas do Brasil, 40).

SANTOS, H.G. dos; JACOMINE, P.K.T.; ANJOS, L.H.C. dos; OLIVEIRA, V.A. de; OLIVEIRA, J.B. de; COELHO, M.R.; LUMBRERAS, J.F.; CUNHA, T.J.F. (Ed.). Sistema brasileiro de classificação de solos. 2.ed. Rio de Janeiro: Embrapa Solos, 2006. $306 \mathrm{p}$.

STORCK, L.; BISOGNIN, D.A.; OLIVEIRA, S.J.R. de. Dimensões dos ensaios e estimativas do tamanho ótimo de parcela em batata. Pesquisa Agropecuária Brasileira, v.41, p.903-909, 2006.

STORCK, L.; OLIVEIRA, S.J.R. de; GARCIA, D.C.; BISOGNIN, D.A. Comprimento e largura do tamanho ótimo da parcela experimental em batata. Ciência Rural, v.35, p.1043-1048, 2005.

VIANA, A.E.S.; SEDIYAMA, T.; CECON, P.R.; LOPES, S.C.; SEDIYAMA, M.A.N. Estimativas de tamanho de parcela em experimentos com mandioca. Horticultura Brasileira, v.20, p.58-63, 2002.

Recebido em 4 de março de 2009 e aprovado em 31 de junho de 2009 